\title{
Fatal outcome arising from use of a sutureless 'corkscrew' epicardial pacing electrode inserted into apex of left ventricle
}

\author{
Romeo J. Vecht, Colin J. Fontaine, and John W. B. Bradfield \\ From the Departments of Cardiology and Pathology, St. Mary's Hospital, Praed Street, London W2
}

A 59-year-old man is described in whom the insertion of an epicardial sutureless 'corkscrew' electrode resulted in fatal ventricular perforation. Fatal myocardial perforation can occur with this electrode and the apex of the left ventricle should never be used as the site of insertion. Necropsy also showed that the transvenous right ventricular electrode, inserted one year previously, had penetrated a tricuspid leaflet. This could have accounted for the ensuing pacing failure.

\section{Case report}

In June 1974, a 58-year-old man presented with fainting spells. $\mathrm{He}$ was hypertensive $(220 / 120$ $\mathrm{mmHg}$ ), the fundi showing irregular arteries with venous nipping. His electrocardiogram indicated an inferior axis deviation, first degree block, complete right bundle-branch block, and deep $\mathrm{Q}$ waves in lead VI. In December of that year he developed complete heart block. A transvenous electrode was introduced without difficulty through a brachiocephalic vein, and satisfactorily positioned within the right ventricle. Pacing was achieved at a threshold of $0.7 \mathrm{~V}$. Nine months later, however, he again complained of dizzy turns. A 24-hour electrocardiographic tape-recording at that time showed periods of asystole, tachycardia, and extrasystoles (Fig. 1). It was assumed that the pacemaker was failing, and as there was no obvious displacement of the transvenous electrode on plain chest radiography, a new pacemaker was implanted into the original subcutaneous pocket. The previous electrode was left in situ. The threshold was again $0.7 \mathrm{~V}$. A subsequent report confirmed that there had been a premature battery depletion of the original pacemaker.

Soon after, he again complained of persistent dizzy spells and further electrocardiograms showed inconstant pacing. At that stage, as the result of the original battery failure was not known, it was thought best in the interest of the patient to replace the entire endocardial by an epicardial system.

In view of the reported number of 'corkscrew' electrodes used, the safety and simplicity of the procedure, and our own uncomplicated experience with this type of epicardial electrode, it was chosen as most suitable for the needs of this patient. His blood pressure on treatment at that time was $140 / 100 \mathrm{mmHg}$. The heart was approached through the fifth intercostal space. Using light pressure, the 'corkscrew' electrode was screwed into the apex of the left ventricle. At $2 \frac{1}{2}$ turns, arterial bleeding was noticed. The electrode was then gently unscrewed

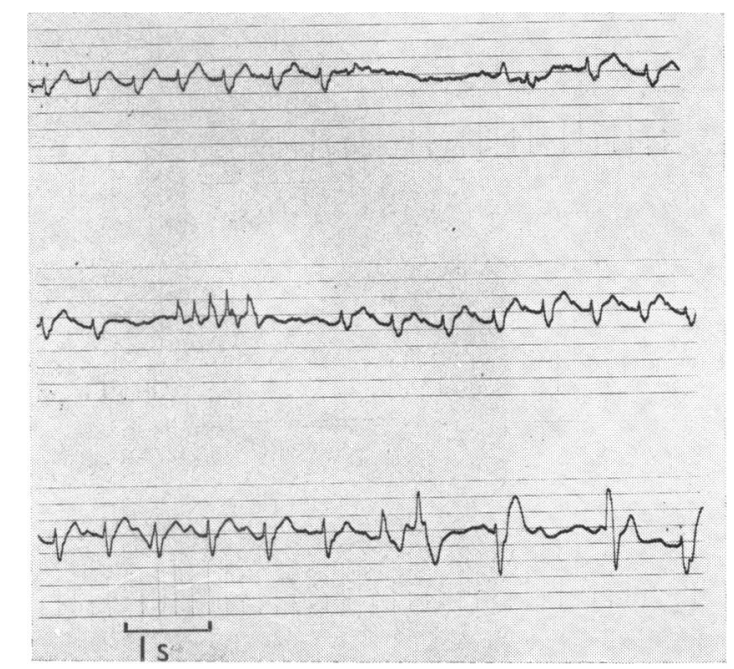

FIG. 1 Non-consecutive strips from a 24-hour electrocardiographic tape-recording showing episodes of asystole, tachycardia, and extrasystoles. 


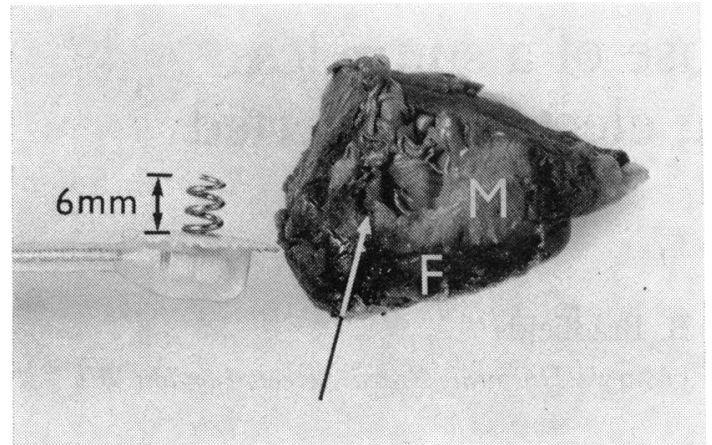

FIG. 2 Necropsy specimen showing apex of the left ventricle situated inferiorly with the 'corkscrew' electrode at same magnification. The arrow marks the path of perforation. F represents epicardial fat, $M$ myocardium.

and the haemorrhage was seen to be caused by ventricular perforation. The bleeding was controlled by means of sutures and buttresses of Teflon felt. Further leakage, however, occurred, which required more extensive buttressing. A similar 'corkscrew' electrode was then implanted uneventfully $4 \mathrm{~cm}$ higher up on the lateral wall of the left ventricle. Ten minutes after closing the wound, there was a dramatic drop in blood pressure because of further rapid haemorrhage. The heart was re-exposed and more suturing was required to control the apical bleeding, but the patient failed to respond to resuscitative measures.
At necropsy the heart was greatly enlarged $(660 \mathrm{~g})$. There was left ventricular hypertrophy with a wall thickness of $1.8 \mathrm{~cm}$ over the outflow tract. At the apex of the left ventricle there was a perforation admitting a finger tip. This was the area where the first electrode had been implanted, and, at that site, the left ventricular wall surprisingly only measured $4 \mathrm{~mm}$ in thickness. There was also a superficial layer of epicardial fat measuring $5 \mathrm{~mm}$.

The electrode itself was slightly longer $(6 \mathrm{~mm})$ than the apical myocardial wall (Fig. 2).

There was no evidence of myocardial infarction or focal fibrosis, though some endocardial fibrosis was noticed on the left aspect of the interventricular septum, and the anterior descending branch of the left coronary artery was narrowed to about 50 per cent of its diameter.

In the right side of the heart, the transvenous electrode had perforated the posteroinferior cusp of the tricuspid valve and was found to slide readily through a channel of organized fibrous tissue (Fig. 3).

\section{Discussion}

The so-called sutureless 'corkscrew' epicardial pacing electrode ${ }^{1}$ has been in use since 1971 (Hunter et al., 1973; Mansour, Fleming, and Hatcher, 1973; Stewart, 1974; Naclerio and Varriale, 1974; Wolner, Stellwag, and Navrátil, 1975). By 1976, approximately 30000 of these had been implanted

${ }^{1}$ Model 6917 Medtronic, Minneapolis, Minnesota.

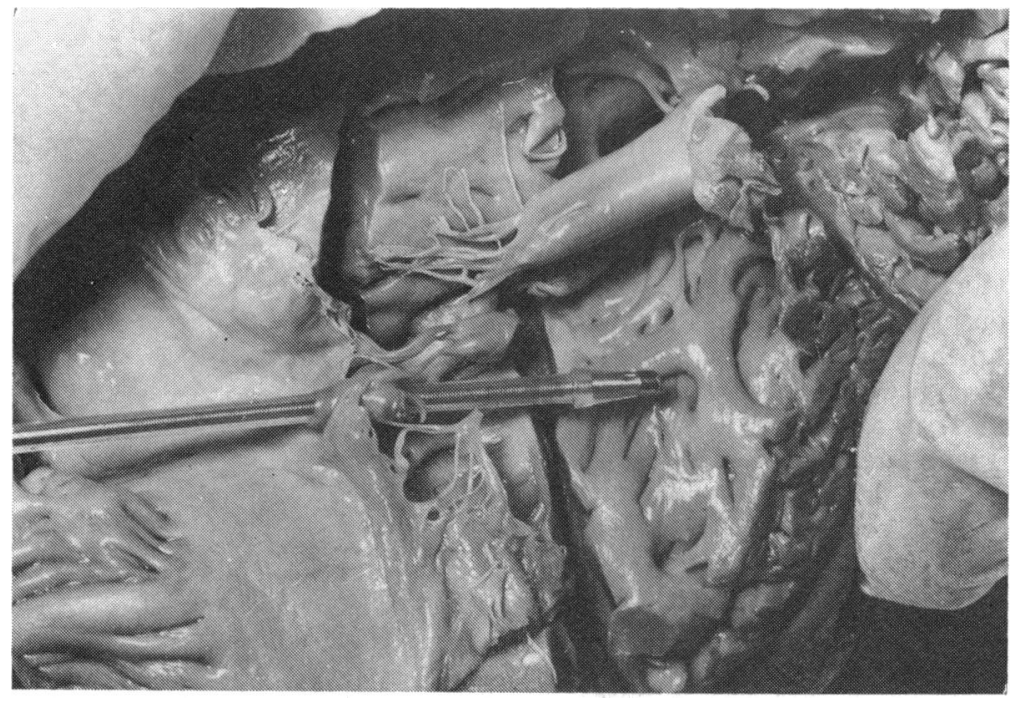

FIG. 3 Necropsy specimen showing transvenous electrode perforating tricuspid leaflet. There is a fibrous tunnel at the site of perforation. 
world wide, 700 in the U.K. To date only 13 mechanical failures caused by fracture of the corkscrew coil have been reported as well as one coductor failure. This is in addition to a small percentage ( 4 to $5 \%$ ) of recognized pacing problems such as exit block, occasional arrhythmias, or infection (J. P. Lawther, Medtronic UK, Slough, Berks, 1976, personal communication). It is generally accepted that complications occur less frequently with epicardial than endocardial systems (McConahay et al., 1971; Dixon et al., 1972; Mansour et al., 1973). The advantages of using the 'corkscrew' type reside in the secure myocardial insertion, in the limited approach and ease of implantation which is well tolerated by patients, in the fact that the subxyphoid insertion can be performed under local anaesthesia, and that the pacing threshold is of a low magnitude.

The site of implantation of the electrode does not appear constant: some authors chose an area as close to the apex of each ventricle as possible (O'Neill and Spagna, 1974). Others suggest a 'healthy looking area of myocardium' without adding further precision (Mansour et al., 1973). Others still recommend the inferior (Stewart, 1974; Roberto, 1974; Wolner et al., 1975) or the anterior surface of the right ventricle (Naclerio and Varriale, 1974). These latter authors also suggest the choice of an area 'free of epicardial fat' whereas Stewart (1974) states that scarred areas of myocardium should be avoided.

Over the years, in the light of clinical trials and increasing experience, the chosen site of insertion has tended to be from the left to the right ventricle, the latter being an easier approach (J. P. Lawther, 1976, personal communication). Mansour reported 3 failures in 26 patients using this technique. One patient died from a myocardial infarction two weeks after operation, another had repeated ventricular tachycardia, and a third had a rising threshold. In Naclerio's series of 36 implantations, there were two deaths, one two months after operation from intractable congestive failure, and one from myocardial infarction after a major orthopaedic procedure. In the 30 cases of Wolner et al., there were several complications. In one patient, the electrode became separated from the myocardium two hours after operation. This was thought to be the result of incomplete implantation and was rectified. One patient developed a high threshold and another an infection at the site of insertion. Two of their patients died, one from ventricular fibrillation and another from pneumonia. Stewart, Cohen, and Murphy (1975) described an instance in which the right ventricle was entered by a similar epicardial electrode, but the situation was controlled by sutur- ing the site of the perforation. To the best of our knowledge, no other cases have been reported to date in which the 'corkscrew' electrode was shown to penetrate a ventricular cavity and lead to the death of the patient.

In a series of 25 hearts exposed, at necropsy, to twisting of the anterior surface of the right ventricle in a variety of areas, in no instance was there penetration of the coil into the ventricular cavity (Naclerio and Varriale, 1974).

Prompted by the above observation, one of us (J.W.B.B.) has recently examined 10 consecutive postmortem hearts. The thickness of the myocardium at the tip of the apex was never greater than $2 \mathrm{~mm}$ (to be published).

The pressure difference which exists between the two ventricles conceivably renders a left ventricular haemorrhage more liable to occur, particularly in the presence of a thin apical myocardium or in association with raised blood pressure. Excessive adipose tissue adds a further hazard by obscuring the state of the underlying myocardium and by making an accidental perforation more difficult to repair.

In view of these findings, and the fatal outcome described, we recommend that the 'corkscrew' electrode should not be inserted into the apex of the left ventricle, nor through abundant fat.

It seems likely that the tricuspid cusp perforation seen in our patient was partially responsible for his pacing failure, as the transvenous electrode could have been intermittently dislodged by the movement of the valve. There was no evidence in this patient, of adhesion to the tricuspid valve secondary to endocarditis (Friedberg and D'Cunha, 1969).

We thank Dr. E. M. M. Besterman and Mr. L. L. Bromley for allowing us to report this case.

\section{References}

Dixon, S. H., Jr., Perryman, R. A., Morris, J. J., Jr., and Young, W. G. (1972). Transmediastinal permanent ventricular pacing: a comparison with the transvenous method. Annals of Thoracic Surgery, 14, 206.

Friedberg, H. D., and D'Cunha, G. F. (1969). Adhesions of pacing catheter to tricuspid valve: adhesive endocarditis. Thorax, 24, 498.

Hunter, S. W., Bolduc, L., Long, V., and Quattlebaum, F. W. (1973). A new myocardial pacemaker lead (sutureless). Chest, 63, 430.

McConahay, D. R., McCallister, B. D., Merideth, J., and Danielson, G. K. (1971). Clinical experiences with permanent demand pacemakers. Mayo Clinic Proceedings, 46, 44.

Mansour, K. A., Fleming, W. H., and Hatcher, C. R., Jr. (1973). Initial experience with a sutureless, screw-in electrode for cardiac pacing. Annals of Thoracic Surgery, 16, 127. 
Naclerio, E. A., and Varriale, P. (1974). 'Screw-in' electrode: new method for permanent ventricular pacing. New York State fournal of Medicine, 74, 2391.

O'Neill, J. E., and Spagna, P. M. (1974). Implantation of the model 6917 sutureless lead wire and subxiphoid supradiaphragmatic approach. Medtronic Inc., Minnępolis, U.S.A.

Roberto, A. E. (1974). In Discussion to Naclerio and Varriale (1974). New York State fournal of Medicine, 74, 2396.

Stewart, S. (1974). Placement of the sutureless epicardial pacemaker lead by the subxiphoid approach. Annals of Thoracic Surgery, 18, 308.
Stewart, S., Cohen, J., and Murphy, G. (1975). Sutureless epicardial pacemaker lead: a satisfactory preliminary experience. Chest, 67, 564.

Wolner, E., Stellwag, F., and Navrátil, J. (1975). Myokardiale Schraubelektrode zur Schrittmacher-therapie. Wiener klinische Wochenschrift, 87, 212.

Requests for reprints to Dr. Romeo J. Vecht, Department of Cardiology, St. Mary's Hospital, London W2 $1 \mathrm{NY}$. 